\title{
WEAK PRODUCTS OF UNIVERSAL ALGEBRAS
}

\author{
ILDIKÓ SAIN \\ Mathematical Institute of the Hungarian Academy of Sciences \\ Budapest, PF. 127, H-1364, Hungary
}

\begin{abstract}
Weak direct products of arbitrary universal algebras are introduced. The usual notion for groups and rings is a special case. Some universal algebraic properties are proved and applications to cylindric and polyadic algebras are considered.
\end{abstract}

1. Introduction. The universal algebraic notions of weak direct products introduced in the literature so far ([10, Def. 1, p. 139], [11, p. 104]) are not really universal algebraic because of the following. In any similarity class $\mathrm{K}$ of algebras, the weak direct products as defined in Grätzer [10] or in [11] do not exist, except in the trivial case when the similarity type of $\mathrm{K}$ consists of a single constant symbol only. As it is explained in [10, Ex. 45, p. 156], neither rings with unit element nor Boolean algebras have weak direct products in the sense of [10] or [11]. At the same time, weak direct products have been introduced ("locally", that is, in a non-universal algebraic fashion) in the literature, for many of those classes of algebras for which the version of the concept introduced in [10] or [11] does not exist. These weak direct products do play an important rôle in the recent literature (see e.g. several sections of [16] or [9]).

Here we suggest an improved (generalized) version of the concept introduced in [10] or [11]. This version of a weak direct product exists in most cases.

(In passing we note that weak direct products are used in algebra not only for obtaining structure theorems as it might be the case in group theory. Weak direct products play an important rôle in algebraic logic, e.g. in Boolean algebra theory - and for Boolean algebras, no structure theorem holds with weak direct products.)

1991 Mathematics Subject Classification: 08B25, 03G15, 08C99.

Key words and phrases: universal algebra, algebraic logic, cylindric algebras.

Research supported by Hungarian National Foundation for Scientific Research grant No. 1911. A preliminary version of the present paper was [21].

The paper is in final form and no version of it will be published elsewhere. 
2. New concept of a weak direct product. Throughout the paper, $t$ stands for a similarity type such that in the similarity class $\mathrm{Alg}_{t}$ of all algebras of type $t$, every algebra has a minimal (i.e. smallest) subalgebra. (There are two ways of achieving this: either $t$ contains at least one constant symbol, or else the empty algebra is not excluded from $\mathrm{Alg}_{t}$. Here we do not care which one is the case.)

Let $\mathfrak{A}_{i} \in \mathrm{Alg}_{t}$ for each $i \in I$, for some set $I$. Then $\underset{i \in I}{\mathbf{P}} \mathfrak{A}_{i}$ denotes the direct product of these algebras in the usual sense (cf. [10] or [11, Def. 0.3.1]).

Definition 1 below generalizes [11, Remark 0.3.60, p. 104]. It is also a generalization of $[10$, p. 139]. Note that the Boolean algebras do not have infinite weak direct products in the sense of [10] or [11], but they do in the sense of our Definition 1. Weak direct products of Boolean algebras proved to be rather useful (see e.g. [16]). The same applies to relation algebras (cf. [9]). Also note that the usual notion for groups and rings is still a special case of the weak direct product in the sense of Definition 1.

Definition 1. Let $\left\langle\mathfrak{A}_{i}: i \in I\right\rangle$ be a system of (similar) algebras of similarity type $t$. The weak direct product $\mathbf{P}_{i \in I}^{\mathbf{w}} \mathfrak{A}_{i}$ of $\left\langle\mathfrak{A}_{i}: i \in I\right\rangle$ is defined as follows.

Let $M$ denote the universe of the minimal subalgebra of $\underset{i \in I}{\mathbf{P}} \mathfrak{A}_{i}$. Then

$$
\underset{i \in I}{\mathbf{P}^{\mathbf{w}}} A_{i} \stackrel{\text { def }}{=}\left\{f \in \underset{i \in I}{\mathbf{P}} A_{i}:(\exists g \in M)(\{i \in I: f(i) \neq g(i)\} \text { is finite })\right\} .
$$

$\underset{i \in I}{\mathbf{P}} \mathfrak{A}_{i}$ is defined to be the subalgebra of $\underset{i \in I}{\mathbf{P}} \mathfrak{A}_{i}$ with universe $\underset{i \in I}{\mathbf{p}} A_{i}$.

Clearly, $\mathbf{P}_{i \in I}^{\mathbf{w}} \mathfrak{A}_{i}$ is unique.

Proposition 1. (i) Definition 1 is correct in the sense that $\underset{i \in I}{\mathbf{w}} A_{i}$ is a subuniverse of $\underset{i \in I}{\mathbf{P}} \mathfrak{A}_{i}$.

(ii) $\underset{i \in I}{\mathbf{P}} \mathfrak{A}_{i}$ is a subdirect product if the minimal subalgebra $M$ of $\underset{i \in I}{\mathbf{P}} \mathfrak{A}_{i}$ is nonempty, e.g. if $t$ contains a constant symbol.

The proof is left to the reader.

Rem ark 1 . We could generalize Definition 1 so that it could have any fixed filter $F$ on $I$ as its parameter. Then the last part of the formula would read as $\{i \in I: f(i)=g(i)\} \in F$.

3. Universal algebraic and model theoretic results. Let $\mathrm{K} \subseteq \mathrm{Alg}$. That is, $\mathrm{K}$ is a class of algebras of similarity type $t . \mathbf{P}^{\mathbf{w}} \mathrm{K}$ denotes the class of (isomorphic copies of) all weak products of possibly infinite families of algebras in $\mathrm{K}$ :

$$
\mathbf{P}^{\mathbf{w}} \mathrm{K} \stackrel{\text { def }}{=} \mathbf{I}\left\{\underset{i \in I}{\mathbf{P}_{i \in}^{\mathbf{w}}} \mathfrak{A}_{i}:\left\{\mathfrak{A}_{i}: i \in I\right\} \text { is a subset of } \mathrm{K}\right\},
$$

where $\mathbf{I}$ is the operator of taking isomorphic copies. $\mathbf{P o}{ }^{\mathbf{w}} \mathrm{K}$ denotes the class of weak powers of elements of $\mathrm{K}$. We shall use the notations HK (homomorphic 
images of algebras in K), SK (subalgebras of algebras in $\mathrm{K}$ ), $\mathbf{P K}$ (products of algebras from $\mathrm{K}$ ) as defined in [10] or [11]. UpK denotes the class of ultraproducts of elements of $\mathrm{K}$ (see [11]). All these are meant up to isomorphisms.

We shall consider $\mathbf{H}, \mathbf{S}, \mathbf{P}, \mathbf{U} \mathbf{p}, \mathbf{P}^{\mathbf{w}}$, and $\mathbf{P} \mathbf{o}^{\mathbf{w}}$ as operators on the class $\mathrm{Alg}_{t}$ of all universal algebras of some fixed similarity type $t$ (see [19], [11, p. 89, above Thm. 0.3.17], [4], [14, p. 387], or [10, p. 152, §23]). Namely, with any class $\mathrm{K} \subseteq \mathrm{Alg}_{t}$, the operator $\mathbf{H}$ correlates another class $\mathbf{H K} \subseteq \mathrm{Alg}_{t}$. Juxtaposition of names of operators denotes composition. For example, HSP is the operator correlating with each $\mathrm{K} \subseteq \mathrm{Alg}_{t}$ the class HSPK (see [11, p. 109] or [10]). The statement "HH $=\mathbf{H} "$ means that for every similarity type $t$ and every class $\mathrm{K} \subseteq \mathrm{Alg}_{t}$ we claim $\mathbf{H H K}=\mathbf{H K}$ (cf. [11, Thm. 0.2.23]). On the other hand, $\mathbf{S H} \neq \mathbf{H} \mathbf{S}$ means that there exists a similarity type $t$ and a class $\mathrm{K} \subseteq \mathrm{Alg}_{t}$ such that $\mathbf{S H K} \neq \mathbf{H S K}$ (cf. [11, 0.2.19] and [19]).

Recall from e.g. [14], [13] or [18, Thm. 3] that HSP and SPUp are the closure operators of generating the smallest variety and generating the smallest subvariety respectively. That is, HSPK and SPUpK are the smallest classes containing K and axiomatizable by equations and equational implications, respectively.

Proposition 2. (0) $\mathbf{P} \neq \mathbf{P}^{\mathbf{w}}$.

(i) $\mathbf{H S U p P}^{\mathbf{w}}=\mathbf{H S P}=\mathbf{H S U p P}, \mathbf{S U p P}^{\mathbf{w}}=\mathbf{S P U p}=\mathbf{S U p P}$.

(ii) $\mathbf{H S P}^{\mathbf{w}} \mathbf{U} \mathbf{p} \neq \mathbf{H S P}, \mathbf{S P}^{\mathbf{w}} \mathbf{U} \mathbf{p} \neq \mathbf{S P U} \mathbf{p}$.

(iii) $\mathbf{H S P}^{\mathbf{w}} \mathrm{K}$ is not first order axiomatizable, for some $\mathrm{K} \subseteq \mathrm{Alg}_{t}$.

(iv) $\mathbf{H S P} \mathbf{P}^{\mathbf{w}}, \mathbf{S P}^{\mathbf{w}}, \mathbf{H} \mathbf{P}^{\mathbf{w}}, \mathbf{P}^{\mathbf{w}}, \mathbf{H S} \mathbf{P}^{\mathbf{w}} \mathbf{U} \mathbf{p}, \mathbf{S P}^{\mathbf{w}} \mathbf{U p}$ are not closure operators, though $\mathbf{H S U} \mathbf{p} \mathbf{P}^{\mathbf{w}}$ and $\mathbf{S U p P} \mathbf{p}^{\mathbf{w}}$ are.

(v) $\mathbf{H S P o}^{\mathbf{w}}$ preserves the formulas of the following shape:

$$
\bigvee_{i<\alpha}\left(\bigwedge_{i \leq j<\alpha} e_{j}\right)
$$

where $\alpha$ is an arbitrary ordinal, and $\left\{e_{j}: j<\alpha\right\}$ is a set of equations which contains a finite set of variables only (i.e., let $\beta$ be a formula of the above shape; then $\mathrm{K} \vDash \beta$ implies $\mathbf{H S P o}^{\mathrm{w}} \mathrm{K} \vDash \beta$ ).

(vi) $\mathbf{S P o}{ }^{\mathbf{w}}$ preserves all the formulas of the following shape:

$$
\bigwedge_{x \in X} e_{x} \rightarrow \bigvee_{i<\alpha}\left(\bigwedge_{i \leq j<\alpha} e_{j}\right)
$$

where $X$ is an arbitrary set; $\alpha$ is an ordinal; $e_{x}, e_{j}$ are equations (of type $\left.t\right)$; and $\left\{e: e\right.$ is $e_{x}$ for some $x \in X$ or $e_{j}$ for some $\left.j<\alpha\right\}$ contains a finite set of variables only.

P r o of. Notation: If $\mathbf{Q}, \mathbf{Q}_{\mathbf{1}}$ are operators, then $\mathbf{Q} \subseteq \mathbf{Q}_{\mathbf{1}}$ means that $\mathbf{Q K} \subseteq$ $\mathrm{Q}_{\mathbf{1}} \mathrm{K}$ for every $\mathrm{K}$ (cf. [19]).

Proof of (i). It is known that HSP $=\mathbf{H S U p P}$ (see e.g. [11, Thm. 0.4.64]). To prove $\mathbf{S U} \mathbf{p} \mathbf{P}^{\mathbf{w}}=\mathbf{S U p P}=\mathbf{S P U p}$ we shall use the following lemma. 
LEMMA 2.1. Let $\mathbf{P}^{\mathbf{f}}$ and $\mathbf{P}^{\mathbf{r}}$ denote the operators of taking all finite products and all reduced products respectively. Let $\mathbf{Q}$ be an operator such that $\mathbf{P}^{\mathbf{f}} \subseteq \mathbf{Q} \subseteq$ $\mathbf{S P}^{\mathbf{r}}$. Then

$$
\text { SUpQ }=\text { SPUp }
$$

Pro of. Some notation: Let $\mathrm{K} \subseteq \mathrm{Alg}_{t}$. Then

$$
\begin{aligned}
& \text { Univ } \mathrm{K} \stackrel{\text { def }}{=}\left\{\left(\bigwedge_{i \in I} e_{i} \rightarrow \bigvee_{j \in J} p_{j}\right): \mathrm{K} \vDash\left(\bigwedge_{i \in I} e_{i} \rightarrow \bigvee_{j \in J} p_{j}\right)\right. \text { and } \\
&\left.\left\{e_{i}: i \in I\right\} \cup\left\{p_{j}: j \in J\right\} \text { is a finite set of equations of type } t\right\}, \\
& \text { Qeq } \mathrm{K} \stackrel{\text { def }}{=}\left\{\left(\bigwedge_{i \in I} e_{i} \rightarrow p\right):\left(\bigwedge_{i \in I} e_{i} \rightarrow p\right) \in \text { Univ } \mathrm{K}\right\} .
\end{aligned}
$$

If $\Sigma$ is a set of formulas, then $\operatorname{Mod}(\Sigma)$ denotes the class of all models of $\Sigma$.

Now, SUpQK = Mod Univ QK, by [11, Thm. 0.3.83] and [18, Thm. 3(v)].

It is not hard to prove that

(*) $\left(\mathbf{P}^{\mathbf{f}} \mathrm{K} \vDash\left(\bigwedge_{i \in I} e_{i} \rightarrow \bigvee_{j \in J} p_{j}\right)\right.$ and $J$ is finite $) \Rightarrow(\exists j \in J) \mathrm{K} \vDash\left(\bigwedge_{i \in I} e_{i} \rightarrow p_{j}\right)$

(see e.g. [18, Lemma 5]).

Now $(*), \mathbf{P}^{\mathbf{f}} \subseteq \mathbf{Q} \subseteq \mathbf{S P}^{\mathbf{r}}$, and the fact that $\mathbf{S P}^{\mathbf{r}}$ preserves quasi-equations (i.e. elements of $Q e q \emptyset$ ) imply that

$$
\text { Mod Univ } \mathbf{Q K}=\operatorname{Mod} Q e q \mathrm{~K} \text {. }
$$

It is known that Mod $Q e q \mathrm{~K}=\mathbf{S P U p K}$ (see e.g. [13], [18, Thm. 3(vi)]). Lemma 2.1 is proved.

Since $\mathbf{P}^{\mathbf{f}} \subseteq \mathbf{P} \subseteq \mathbf{S P}^{\mathbf{r}}$ and $\mathbf{P}^{\mathbf{f}} \subseteq \mathbf{P}^{\mathbf{w}} \subseteq \mathbf{S P}^{\mathbf{r}}$, Lemma 2.1 implies $\mathbf{S U p} \mathbf{P}^{\mathbf{w}}=$ $\mathbf{S U p P}=\mathbf{S P U p}$. Thus (i) is proved.

Proof of (0), (ii), and (iv). To prove (0), (ii), and (iv) it is enough to prove

$$
\mathbf{H S P}^{\mathrm{w}} \mathbf{U} \mathbf{p} \nsupseteq \mathbf{P} \text { and } \quad \mathbf{H S P}^{\mathrm{w}} \mathbf{U} \mathbf{p} \nsupseteq \mathbf{P}^{\mathbf{w}} \mathbf{P}^{\mathbf{w}} .
$$

We shall fix a class $\mathrm{K}$ of algebras for which

$$
\mathbf{H S P}^{\mathrm{w}} \mathbf{U p K} \nsupseteq \mathbf{P K} \text { and } \mathbf{H S P}^{\mathrm{w}} \mathbf{U} \mathbf{p K} \nsupseteq \mathbf{P}^{\mathrm{w}} \mathbf{P}^{\mathrm{w}} \mathrm{K} \text {. }
$$

Let the similarity type $t$ be $t \stackrel{\text { def }}{=}\left\{(0,0),(1,0),\left(f_{i}, 1\right),\left(g_{i}, 1\right): i \in \omega\right\}$. Now

$$
\begin{aligned}
& \mathrm{K} \stackrel{\text { def }}{=}\left\{\mathfrak{A} \in \operatorname{Mod}_{t}:\right. \\
& \left.\qquad A=\{0,1\} \text { and for every } i \in \omega, \mathfrak{A} \vDash f_{i} 0=0 \text { and } \mathfrak{A} \vDash g_{i} 1=1\right\} .
\end{aligned}
$$

LEMma 2.2. For every element "a" of an arbitrary algebra $\mathfrak{A} \in \mathbf{H S P}{ }^{\mathbf{w}} \mathbf{U} \mathbf{p K}$, either $\left\{f_{i} a: i \in \omega\right\}$ is finite or $\left\{g_{i} a: i \in \omega\right\}$ is finite.

Proof. It is enough to prove the lemma for every $\mathfrak{A} \in \mathbf{P}^{\mathbf{w}} \mathrm{K}$, since the operator HS "preserves" the above property, and UpK $=$ K. Let $\mathfrak{A}=\underset{i \in I}{\mathbf{P}} \mathfrak{A}_{i}$ and 
$\left\{\mathfrak{A}_{i}: i \in I\right\} \subseteq \mathrm{K}$. Let $a \in A, a=\left\langle a_{i}: i \in I\right\rangle$. Now, either $\left\{i \in I: a_{i} \neq 0\right\}$ is finite or $\left\{i \in I: a_{i} \neq 1\right\}$ is finite. Now, $\mathrm{K} \vDash\left\{f_{i} 0=0, g_{i} 1=1: i \in \omega\right\}$ completes the proof of Lemma 2.2.

Next we define a system $\left\langle\mathfrak{A}_{i}: i \in \omega+\omega\right\rangle$ of algebras of K. Let $i, j \in \omega$. In the algebra $\mathfrak{A}_{i}$ we define the operations $f_{j}$ and $g_{j}$ as follows:

$$
f_{j}(1)=\left\{\begin{array}{ll}
0 & \text { if } j \leq i, \\
1 & \text { otherwise, }
\end{array} \quad g_{j}=\text { Identity } .\right.
$$

In $\mathfrak{A}_{\omega+i}$ we define $f_{j}$ and $g_{j}$ as follows:

$$
g_{j}(0)=\left\{\begin{array}{ll}
1 & \text { if } j \leq i, \\
0 & \text { otherwise, }
\end{array} \quad f_{j}=\text { Identity } .\right.
$$

Let $\mathfrak{A}^{\prime}=\underset{i \in \omega+\omega}{\mathbf{P}} \mathfrak{A}_{i}$ and $\mathfrak{A}_{1}^{\prime}=\underset{i \in \omega}{\mathbf{w}} \mathfrak{A}_{i} \times \mathbf{P}_{i \in \omega}^{\mathbf{w}} \mathfrak{A}_{\omega+i}$. Then $\mathfrak{A}^{\prime} \in \mathbf{P K}, \mathfrak{A}_{1}^{\prime} \in \mathbf{P}^{\mathbf{w}} \mathbf{P}^{\mathbf{w}} \mathrm{K}$, and $\mathfrak{A}_{1}^{\prime} \subseteq \mathfrak{A}^{\prime}$. For the element $a^{\prime}=\langle 0,0, \ldots, 1,1, \ldots\rangle=A_{1}^{\prime}$ neither $\left\{f_{i} a^{\prime}: i \in \omega\right\}$ nor $\left\{g_{i} a^{\prime}: i \in \omega\right\}$ is finite (both in $\mathfrak{A}_{1}^{\prime}$ and $\mathfrak{A}^{\prime}$ ). Thus, by Lemma 2.2 , neither $\mathfrak{A}^{\prime}$ nor $\mathfrak{A}_{1}^{\prime}$ is in $\mathbf{H S P}^{\mathbf{w}} \mathbf{U p K}$, proving $\mathbf{H S P} \mathbf{P}^{\mathbf{w}} \mathbf{U p} \nsupseteq \mathbf{P}$ and $\mathbf{H S P}^{\mathbf{w}} \mathbf{U} \mathbf{p} \nsupseteq \mathbf{P}^{\mathbf{w}} \mathbf{P}^{\mathbf{w}}$. So, (0), (ii), and (iv) are proved.

Proof of (iii). Recall from [11] that $\mathrm{Lf}_{\omega}$ denotes the class of all locally finitedimensional cylindric algebras. $\mathbf{H S P}^{\mathbf{w}} \mathrm{Lf}_{\omega}=\mathrm{Lf}_{\omega}$ but $\mathrm{Lf}_{\omega}$ is not axiomatizable.

Proof of (vi). Let $\beta=\left(\bigwedge_{x \in X} e_{x} \rightarrow \bigvee_{i<\alpha}\left(\bigwedge_{i \leq j<\alpha} e_{j}\right)\right)$ be a formula of the required shape, and let $\left\{v_{1}, \ldots, v_{m}\right\}$ be the set of variables occurring in $\beta$. Let $\mathfrak{A} \vDash \beta$. We have to prove

$$
\underset{i \in I}{\mathbf{P}^{\mathbf{w}}} \mathfrak{A}_{i}=\mathfrak{A}^{\prime} \vDash \beta,
$$

where $\mathfrak{A}_{i}$ is $\mathfrak{A}$ for every $i \in I$. Suppose that

$$
\mathbf{P}_{i \in I}^{\mathbf{w}} \mathfrak{A}_{i} \vDash\left(\bigwedge_{x \in X} e_{x}\right)\left[a_{1}, \ldots, a_{m}\right] .
$$

For every projection function $p j_{i}$, we denote $p j_{i}\left(a_{r}\right)$ by $a_{r}(i)$. We then have $\mathfrak{A} \vDash$ $\left(\bigwedge_{x \in X} e_{x}\right)\left[a_{1}(i), \ldots, a_{m}(i)\right]$. Then, since $\mathfrak{A} \vDash \beta$, we have

$$
\mathfrak{A} \vDash \bigvee_{z \in \alpha}\left(\bigwedge_{z_{i} \leq j<\alpha}\right)\left[a_{1}(i), \ldots, a_{m}(i)\right] .
$$

Thus for every $i \in I$, there exists $z_{i} \in \alpha$ such that

$$
\mathfrak{A} \vDash\left(\bigwedge_{z_{i} \leq j<\alpha} e_{j}\right)\left[a_{1}(i), \ldots, a_{m}(i)\right],
$$

that is, such that

$$
\mathfrak{A} \vDash\left\{e_{j}: z_{i} \leq j<\alpha\right\}\left[a_{1}(i), \ldots, a_{m}(i)\right] .
$$


Since $\mathfrak{A}_{i}$ is $\mathfrak{A}$ for every $i \in I$ and $a_{1}, \ldots, a_{m} \in \underset{i \in I}{\mathbf{P}} A_{i}$, there is a finite $J \subseteq I$ such that

$$
\left\{\left\langle a_{1}(i), \ldots, a_{m}(i)\right\rangle: i \in I\right\} \subseteq\left\{\left\langle a_{1}(i), \ldots, a_{m}(i)\right\rangle: i \in J\right\} .
$$

Let $r$ be the greatest element of $\left\{z_{i}: i \in I\right\}$ (it exists since $J$ is finite). Then

$$
\mathfrak{A} \vDash\left\{e_{j}: r \leq j<\alpha\right\}\left[a_{1}(i), \ldots, a_{m}(i)\right],
$$

for every $j \in J$, and therefore also for every $i \in I$. This implies

$$
\underset{i \in I}{\mathbf{P}} \mathfrak{A}_{i} \vDash\left\{e_{j}: r \leq j<\alpha\right\}\left[a_{1}, \ldots, a_{m}\right],
$$

since subalgebras and direct products preserve equations and $\mathbf{P}^{\mathbf{w}} \subseteq \mathbf{S P}$. Therefore

$$
\underset{i \in I}{\mathbf{P}^{\mathbf{w}}} \mathfrak{A}_{i} \vDash\left(\bigwedge_{x \in X} e_{x} \rightarrow \bigvee_{z<\alpha}\left(\bigwedge_{z \leq j<\alpha} e_{j}\right)\right)\left[a_{1}, \ldots, a_{m}\right] .
$$

Since $a_{1}, \ldots, a_{m}$ were arbitrary, (vi) is proved.

Proof of (v). This is a consequence of (vi) and the fact that $\mathbf{H}$ preserves positive formulas even if they are infinitary.

The proof of Proposition 2 is complete.

Remark 2. Properties of the operator $\mathbf{H S P}^{\mathbf{f}}$ were investigated in [7] and $[20]$.

Recall that if $\mathrm{K}$ contains finite algebras only, then $\mathbf{P K}$ contains no countable algebras.

Proposition 3. Let $t$ contain a constant symbol. Let $\alpha^{\prime}$ be an infinite cardinal such that $(\exists \mathfrak{A} \in \mathrm{K}) 1<|A| \leq \alpha^{\prime}$. Then $\mathbf{P}^{\mathbf{w}} \mathrm{K}$ contains an algebra of cardinality $\alpha^{\prime}$.

Proof. Let $\mathfrak{A} \in \mathrm{K}$ be such that $1<|A| \leq \alpha^{\prime}$. Let $\mathfrak{A}^{\prime}=\underset{i \in \alpha}{\mathbf{P}} \mathfrak{A}$. Now, $\left|A^{\prime}\right|=\alpha^{\prime}$, because $|A| \geq 2$ and $\alpha^{\prime} \cdot \alpha^{\prime}=\alpha^{\prime}$ (since $\alpha^{\prime}$ is infinite).

\section{Examples and applications to cylindric algebras}

ExAmples. 1. Weak direct products of Boolean algebras have been studied recently in [16], [15], see also [6, p. 20, above Question 50].

2. In discussions of various special classes of algebras, in particular in the theories of groups and rings, weak products actually play a more important rôle than ordinary direct products (cf. e.g. [11, p. 105]).

3. $\mathbf{P}^{\mathbf{w}}$ is specially important for cylindric algebras, because $\mathbf{P}^{\mathbf{w}} \mathbf{L f}_{\alpha}=\mathrm{Lf}_{\alpha}$, moreover, $\mathbf{H S P}^{\mathbf{w}} \mathrm{Lf}_{\alpha}=\mathrm{Lf}_{\alpha}$, and the class $\mathrm{Lf}_{\alpha}$ is the class of all first order theories when considered as algebras (see [2, Thm. 5.3] and [1, V.5, VI.5]).

Proposition 4. $\mathbf{P}^{\mathbf{w}} \mathrm{Lf}_{\alpha}=\mathrm{Lf}_{\alpha}$.

P r o of. Let $\mathfrak{A}_{i} \in \mathrm{Lf}_{\alpha}$ for every $i \in I$. Let $f \in \underset{i \in I}{\mathbf{w}} A_{i}$ be arbitrary. By Definition 1 , there is a $g \in M \subseteq \underset{i \in I}{\mathbf{P}} A_{i}$, where $M$ is the minimal subalgebra of $\underset{i \in I}{\mathbf{P}} \mathfrak{A}_{i}$, such that $f$ and $g$ differ only at finitely many places, that is, $\{i \in I: f(i) \neq$ 
$g(i)\}$ is finite. By [11, Thm. 2.4.2], we have $\Delta f=\bigcup\{\Delta f(i): i \in I\}$. Also, $(\forall i \in I) \Delta g(i) \subseteq \Delta g$ and $\Delta g$ is finite by [11, Thm. 2.1.16]. Since $(\forall i \in I)[\Delta f(i)$ is finite] by $\mathfrak{A}_{i} \in \mathrm{Lf}_{\alpha}$, we can conclude that also $\Delta f$ is finite.

Problem 1 (cf. [11]). Let SiLf denote the class of simple elements of $L f_{\omega}$. Obviously, $\mathbf{H S P}^{\mathrm{w}} \mathrm{SiLf} \subseteq \mathrm{Lf}_{\omega}$. Is $\mathbf{H S P}^{\mathrm{w}} \mathrm{SiLf}=\mathrm{Lf}_{\omega}$ also true?

The importance and basic properties of the class SiLf were discussed in [1], [2], [3], [12], and in [17].

Continuation of Examples. 4. Weak direct sum of vector spaces is a special case of weak direct product $\mathbf{P}^{\mathbf{w}}$ as defined here (see [5, p. 42]).

5. Direct sums of modules are also a special case of weak direct products, as are direct sums of Abelian groups (see e.g. [8]).

Recall that for groups, rings, semigroups with zero (annihilator), $\mathbf{P}^{\mathbf{w}} \mathbf{P}^{\mathbf{w}}=\mathbf{P}^{\mathbf{w}}$ (see also Proposition 5(i) below).

Proposition 5. (i) Let $\mathbf{V}=\mathbf{P}^{\mathbf{w}} \mathbf{V}$ be a class of algebras in which the oneelement algebra is initial (that is, every algebra in $\mathrm{V}$ contains a minimal subalgebra and the minimal subalgebra has exactly one element). Then in $\mathrm{V}$ we have $\mathbf{P}^{\mathbf{w}} \mathbf{P}^{\mathbf{w}}=\mathbf{P}^{\mathbf{w}}$, that is, for every $\mathrm{K} \subseteq \mathrm{V}$ we have $\mathbf{P}^{\mathbf{w}} \mathbf{P}^{\mathbf{w}} \mathrm{K}=\mathbf{P}^{\mathbf{w}} \mathrm{K}$. ators.

(ii) For Boolean algebras, $\mathbf{P}^{\mathbf{w}}, \mathbf{S} \mathbf{P}^{\mathbf{w}}, \mathbf{S} \mathbf{P}^{\mathbf{w}} \mathbf{U} \mathbf{p}, \mathbf{P}^{\mathbf{w}} \mathbf{U p}$ are not closure oper-

(iii) For rings $\langle R ;+, \cdot, 0,1\rangle$ with unit, (ii) holds.

Proof. The proof of (i) is left to the reader.

Proof of (ii). Let $\underline{2}=\langle 2 ; \cap, \cup, \backslash\rangle$ denote the two-element Boolean algebra, and $\mathrm{K}=\{\underline{2}\}$. Let $\mathfrak{A}^{\prime} \in \mathbf{S P}^{\mathbf{w}} \mathbf{K}$ be arbitrary. Then $\left(\forall a^{\prime} \in A^{\prime}\right)\left[\left\{x \in A^{\prime}: x>a^{\prime}\right\}\right.$ is finite or $\left\{x \in A^{\prime}: x<a^{\prime}\right\}$ is finite]. But this is not true for elements of $\mathbf{P}^{\mathbf{w}} \mathbf{P}^{\mathbf{w}} \mathrm{K}$ : Let $\mathfrak{A}^{\prime}=\mathbf{P}_{i \in \omega}^{\mathbf{w}} \underline{2}$; then $\left(\exists a^{\prime} \in A^{\prime} \times A^{\prime}\right)\left[\left\{x \in A^{\prime} \times A^{\prime}: x>a^{\prime}\right\}\right.$ is infinite and $\left\{x \in A^{\prime} \times A^{\prime}: x<a^{\prime}\right\}$ is infinite]. (Of course, $<$ is understood in $\mathfrak{A}^{\prime} \times \mathfrak{A}^{\prime}$.) Clearly, $\mathrm{K}=\mathbf{U} \mathbf{p K}$ and thus $\mathbf{S} \mathbf{P}^{\mathbf{w}} \mathbf{U} \mathbf{p K}=\mathbf{S P}^{\mathbf{w}} \mathrm{K}$.

Proof of (iii). Let $\underline{2}=\langle\{0,1\} ;+, \cdot, 0,1\rangle$ be the ring with unit 1 defined by $1+1=0$ (this is the two-element Boolean ring). Let $\leq$ be defined as: $x \leq y$ iff $x \cdot y=x$. Then the proof given for (ii) works by taking $\mathrm{K}=\{\underline{2}\}$.

Problem 2. Find a category theoretic characterization of weak direct products.

\section{References}

[1] H. Andréka, T. Gergely and I. Németi, Purely algebraic construction of first order logics, preprint of Central Research Institute of Hung. Acad. Sci., Budapest, No. KFKI73-71 (1973), 46 pp. 
[2] H. Andréka, T. Gergely and I. Németi, On universal algebraic construction of logics, Studia Logica 36 (1-2) (1977), 9-47.

[3] H. Andréka and I. Németi, A simple, purely algebraic proof of the completeness of some first order logics, Algebra Universalis 5 (1975), 8-15.

[4] - - - Formulas and ultraproducts in categories, Beiträge Algebra Geom. 8 (1979), 133151.

[5] M. A. Arbib and E. G. Manes, Arrows, Structures and Functors: The Categorial Imperative, Academic Press, 1975.

[6] E. K. Van Deuven, J. D. Monk and R. Matatyahu, Some questions about Boolean algebras, preprint, Univ. of Colorado, Boulder, Co., 1979.

[7] S. Eilenberg and M. P. Schützenberger, On pseudovarieties, Adv. in Math. 19 (3) (1976), 413-418.

[8] L. Fuchs, Infinite Abelian Groups, Academic Press, 1970.

[9] S. Givant, The structure of relation algebras generated by relativizations, preprint, Dept. of Math., Mills College, Oakland, Cal., 1991, 152 pp.

[10] G. Grätzer, Universal Algebra, second ed., Springer, Berlin 1979.

[11] L. Henkin, J. D. Monk and A. Tarski, Cylindric Algebras, North-Holland, Amsterdam 1971 and 1985.

[12] L. Henkin, J. D. Monk, A. Tarski, H. Andréka and I. Németi, Cylindric Set Algebras, Lecture Notes in Math. 883, Springer, Berlin 1981.

[13] I. Malcev, Algebraic Systems, Akademie-Verlag, Berlin 1973.

[14] J. D. Monk, Mathematical Logic, Graduate Texts in Math. 37, Springer, Berlin 1978.

[15] - On depth of Boolean algebras, lecture at the Math. Inst. Hungar. Acad. Sci., Budapest, December 1978.

[16] J. D. Monk and R. Bonnet (eds.), Handbook of Boolean Algebras, I-II-III, NorthHolland, Amsterdam 1989.

[17] I. Németi, Connections between cylindric algebras and initial algebra semantics of CF languages, in: Mathematical Logic in Computer Science (Proc. Coll. Salgótarján 1978), B. Dömölki and T. Gergely (eds.), Colloq. Math. Soc. J. Bolyai 26, North-Holland, Amsterdam 1981, 561-605.

[18] I. Németi and I. Sain, Cone-implicational subcategories and some Birkhoff-type theorems, in: Universal Algebra (Proc. Coll. Esztergom 1977), Colloq. Math. Soc. J. Bolyai 29, NorthHolland, Amsterdam 1982, 535-578.

[19] D. Pigozzi, On some operations on classes of algebras, Algebra Universalis 2 (1972), 346-353.

[20] J. Rosický, Concerning equational categories, in: Universal Algebra (Proc. Coll. Esztergom 1977), Colloq. Math. Soc. J. Bolyai 29, North-Holland, Amsterdam 1982.

[21] I. Sain, Weak products for universal algebra and model theory, Diagrammes 8 (1982), S1-S15. 